\title{
Focal epilepsy in India with special reference to lesions showing ring or disc-like enhancement on contrast computed tomography
}

\author{
R S WADIA, C N MAKHALE, A V KELKAR, K B GRANT \\ From the Neurology Clinic, Ruby Hall Clinic and Sassoon General Hospital Pune, and The Poona Medical \\ Centre, Pune, India
}

SUMMARY In 150 consecutive cases of simple partial epilepsy significant CT abnormalities were found in $68 \%$. The commonest lesion noted was a hypodense lesion on unenhanced scan, with a ring or disc-like enhancement on contrast scan, and surrounding hypodensity. This lesion was seen in $39^{\mathrm{C}}$ cases and was more common in patients below the age of 15 years and in those with shorter duration? of fits ( $<6$ months). Nineteen of these cases had focal signs, 16 showed focal slow activity on EEG $\overrightarrow{\vec{\omega}}$ and 17/39 had neither signs nor focal slowing on EEG. Ten cases with a ring or disc enhancing $\stackrel{5}{S}$ lesion had evidence of tuberculosis elsewhere in the body, three more had a past history of tuber- $\frac{3}{3}$ culosis and four others had a history of close contact with a case of tuberculosis. After 3 months of ${ }_{i}^{\circ}$ antitubercular treatment, 23 out of 25 patients who were rescanned showed clearing of the lesion. The two who did not were operated upon, and the lesion was shown histologically to be a tuber ${ }_{0} . \vec{\circ}$ culoma. Ten other cases have done well, but have not been rescanned. Only one case was not treated $\vec{N}$ with antitubercular therapy. She developed fits, altered consciousness, and meningitis and recovere $\infty$ from this serious illness after starting antitubercular therapy. Though not histologically verified, $\$$ seems justified to conclude that in India a ring or disc enhancing lesion is the commonest accorp $\overrightarrow{0}$ paniment of focal epilepsy, and that at least one third (and probably more) of these lesions ane tuberculomas.

Focal epilepsy is a common variety of epilepsy and is usually divided into simple and complex partial seizures. ${ }^{1}$ The former when studied by CT have shown abnormalities in $50-70 \%$ of cases. ${ }^{2-7}$ Those with complex partial seizures show such changes less frequently. ${ }^{2}$ There have been no reports of focal epilepsy studied by CT from India and reports from other oriental countries are few.

We report our findings in a consecutive series of 150 cases, all of them studied by CT and EEG. On CT we have often seen a hypodense lesion on plain scan with ring or disc-like enhancement on contrast scan. Such a lesion has only rarely been reported in the Western literature.

\section{Materials and methods}

One hundred and fifty cases of simple partial epilepsy seen in

Address for reprint requests: Dr R S Wadia, Department of Medicine, Poona Medical Foundation, 40 Sassoon Road, Poona 41001, India.

Received 3 January 1986 and in final revised form 15 December 1986. Accepted 27 January 1987 the Neurology department of the Ruby Hall Clinic, Punêㄹ were chosen for this study. Cases were included if they had $\infty$ simple partial seizures, with CT and EEG studies. During this period 12 cases of simple partial epilepsy were excluded because the CT was not performed, either because the aetiology was obvious (such as stroke, childhood hemiplegia) or because the patient was not willing to have further investigations.

\section{Results}

The 150 cases included 90 males and 60 females, and the age range was 3-77 years. Forty four cases were below 15 years of age and 50 were between 20-40 years. The lesions diagnosed in the study are shown in table 1. The lesion recorded here as ring or disc enhancing lesion consisted of a hypodense focal area on CT scan which on enhancement became a ring or disc with surrounding focal hypodensity (figs 1 and 2 ). This rarely produced distortion of neighbouring 을 structures; it was more common in children, being found in $40.9 \%$ of 44 cases below 15 years, and in $19 \%$ of 106 cases above that age. This difference is significant $(p<0.02)$. A ring or disc enhancing lesion 0 
Table 1 CT scan findings in focal epilepsy

\begin{tabular}{llll}
\hline & $\begin{array}{l}\text { Age below } \\
\text { I5 years } \\
(\%)\end{array}$ & $\begin{array}{l}\text { Age above } \\
\text { 15 years } \\
(\%)\end{array}$ & $\begin{array}{l}\text { Total } \\
(\%)\end{array}$ \\
\hline Total & $44(100)$ & $106(100)$ & $150(100)$ \\
Normal & $14(31 \cdot 8)$ & $34(32 \cdot 1)$ & $48(32)$ \\
Focal disc or ring & $18(40 \cdot 9)$ & $21(19 \cdot 0)$ & $39(26)$ \\
$\quad$ enchancing lesions & $1(2 \cdot 3)$ & $12(11 \cdot 3)$ & $13(9 \cdot 5)$ \\
Tumour & $2(4 \cdot 6)$ & $23(21 \cdot 7)$ & $25(16 \cdot 5)$ \\
Vascular & $1(2 \cdot 3)$ & $4(3 \cdot 75)$ & $5(3 \cdot 3)$ \\
Focal calcification & $1(2 \cdot 3)$ & $9(8 \cdot 4)$ & $10(6 \cdot 6)$ \\
Atrophy & $7(15 \cdot 9)$ & $3(2 \cdot 8)$ & $10(6.6)$ \\
Miscellaneous & & & \\
\hline
\end{tabular}

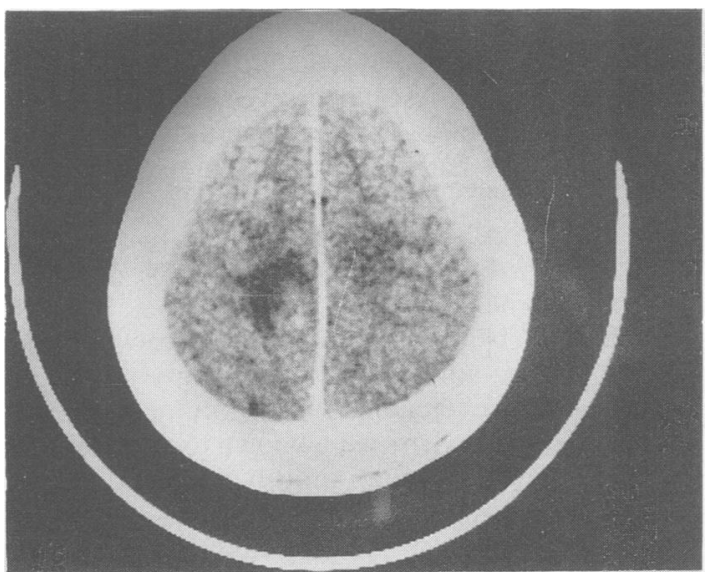

Fig 1 Plain scan of a ring or disc enhancing lesion showing focal hypodense lesion.

was also more common in those with fits of less than 6 months duration ( 32 of 105 cases) than in cases of 6-12 months duration ( 3 of 25 cases). This difference just fails to reach significance $0 \cdot 1>\mathrm{p}>0.05$.

CT scan abnormalities were found in $60.2 \%$ of cases without any signs and in $58.8 \%$ of cases with no EEG abnormality. In both these circumstances ring or disc enhancing lesion was the commonest abnormality. Table 2 compares this series of focal epilepsy with 100 cases from the same hospital (under treatment in the same unit) in the era before CT became available in February 1982. In the older series only 52 were submitted to angiography or pneumoencephalography and the major difference in the groups is that ring or disc enhancing lesions were not diagnosed in the prescan era. Even at the present time some cases of ring or disc enhancing lesion have had an angiogram and no abnormality had been detected.

In 32 cases with ring or disc enhancing lesion the fits were pure motor and in seven were sensory motor. Only 10 lost consciousness during the fit. Twenty seven had had fits for only 3 months or less and five between 3-6 months. Twenty one had shown a sudden cluster of fits. Focal signs were seen in 19 cases.

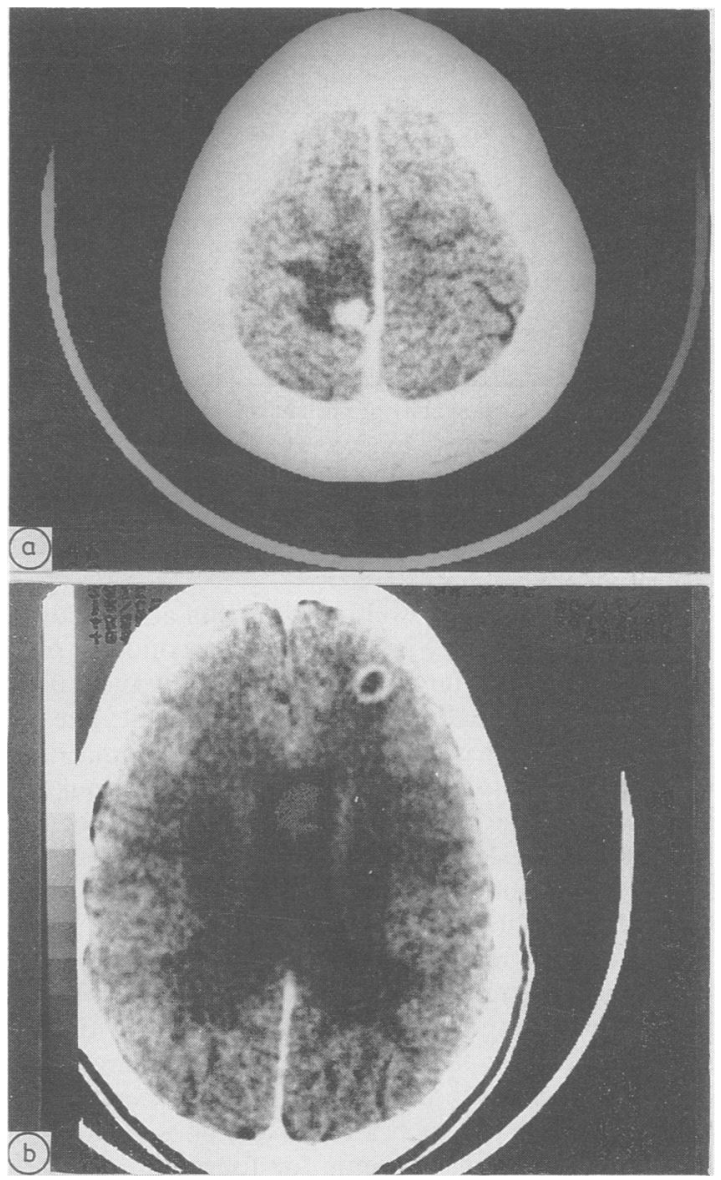

Fig 2 (a) Constrast study of case in fig 1, disc enhancing lesion, (b) contrast study showing ring enchancing lesion.

Sixteen showed focal slowing on EEG and nine showed focal spikes. Of these 39 cases with ring or disc enhancing lesion 10 had evidence of tuberculous infection in the lungs or elsewhere at the time of fits and three had prior history of tuberculosis. Two siblings in one family had the same lesion and their grandfather who was in regular contact with them had died of tuberculosis. There were two others with close contact with a case of tuberculosis. The CSF was abnormal in 4 of 39 cases.

As a tuberculoma is known to have these CT scan appearances ${ }^{8}$ and because of the above association these 39 cases were all treated with standard antituberculous therapy with isomazid, rifampicin and ethambutol/or streptomycin. The course of the disease in these cases was as follows:

(a) Of 25 cases followed up by a second scan after 3 months or longer 23 have cleared completely.

(b) The 2 cases who had not cleared on scanning 
Table 2 Focal epilepsy, before and after availability of CT

\begin{tabular}{lcc}
\hline & CT scan & Prescan \\
\hline Total cases & 150 & $100^{*}$ \\
No cause detected & $32 \%$ & $75 \dagger$ \\
Tumours & $9 \cdot 5 \%$ & 7 \\
Vascular & $16.5 \%$ & $6 \dagger$ \\
Atrophic lesions & $6.6 \%$ & 4 \\
Focal calcification & $3.3 \%$ & 0 \\
Tuberculoma (histologically & $1 \cdot 3 \%$ & 2 \\
verified) & $26 \%$ & $0 \dagger$ \\
Focal ring or disc & $6.6 \%$ & $6 \%$ \\
Other causes & & \\
\hline
\end{tabular}

*52 submitted to angiography or pneumoencephalography. †Significant difference.

after 3 months have been operated upon, and in both a diagnosis of tuberculoma was made histologically.

(c) Ten patients are well with no fits and no other symptoms. They have refused a second CT scan.

(d) In one case after 2 months of therapy the patient developed headache and further deficit. She has been operated upon and an astrocytoma diagnosed. (In table 2 she is reported as tumour and is not included in the 39 cases with ring or disc enhancing lesion). Before the operation a diagnosis of tumour was suspected on the basis of her deterioration.

(e) As recent reports have pointed out that some of these cases of ring or disc enhancing lesion may show disappearance of CT findings with time ${ }^{9}$ it was decided to treat some with anticonvulsants alone, and not give anti-tubercular therapy.

The patients for such a trial were chosen carefully as they would have to come for follow up regularly and agree to repeat CT. A police constable's wife was chosen as the first case. She did come for follow up for 6 weeks and then developed fits, unconsciousness and hemiplegia. She was shown to have meningitis. Her condition was serious for some days and it was decided to start anti-tuberculous treatment. She thereafter made a slow recovery. She had had a repeat scan recently after 9 months therapy. The ring or disc enhancing lesion has resolved. No further trial without antituberculous therapy has been undertaken in this series.

\section{Discussion}

Several series of patients with focal epilepsy studied by CT have been reported in the Western literature. ${ }^{2-7}$ None of these have described the ring or disc enhancing hypodense lesion, though the rate of positive findings is similar to ours. In three of these series $^{237}$ all the abnormalities in patients with focal epilepsy were described. In 154 cases of partial epilepsy in these three series there were 79 focal lesions but no lesion similar to ring or disc enhancing lesion. However, it is known that in India these lesions are seen not infrequently.

Tandom and Bhargava ${ }^{8}$ first reported these lesions as being tuberculomas on the basis of histology in some cases and association with tuberculous meningitis in several others. Hence antituberculous treatment was given in these cases. Subsequently Sethi et al ${ }^{9}$ showed that some lesions may disappear on repeat scanning 3 months later while the patient is on anticonvulsants alone. Sethi et $_{\text {al }}{ }^{9}$ thought these may represent focal encephalitis and Tandon etal (personal communication) suggested that some may represent postictal changes. We have rarely seen these lesions in cases of generalised epilepsy, or in late onset epilepsy. We have rescanned seven patients for various reasons 1-2 months after the initial scan and seen that the lesions had regressed but not disappeared, implying that the regression of these lesions is a gradual and relatively slow process (fig 3 ). In most cases presenting with fits, CT was generally performed 8 days after the fit or later.

Sethi et $\mathrm{l}^{9}$ believe that the disappearance of these lesions without anti-tuberculous therapy proves that these are not tuberculomas. Tuberculous lesions in the lungs and elsewhere were known to clear by them selves in the days before antituberculous drugs and the primary lesion in the lung may clear leaving nof significant clinical or radiological trace in the vas majority of Indians. A relevant finding may be that some cases showed one or two areas of focal calcification as the only abnormality on CT scan These we believe represent healed granulomas. Sethi et al ${ }^{9}$ suggest that these lesions should be watched without antitubercular treatment for 6-12 weeks, and if they persist one is justified in starting antituberculous therapy. We feel that if there was no evidence that they were tubercular to begin with, there persistance is no proof of their tubercular nature. We feel in our 39 cases the two histologically proven, the 10 with present tuberculosis, and the patient treated only with anticonvulsants were certainly tubercular ( 13 cases, $33 \%$ ). Of the remaining 26 cases we have no proof except to point out that they were clinically and radiologically identical to the above 13 , and some had a history of close contact with tuberculosis. It is also to be noted that in all forms of extra pulmonary tuberculosis some cases do not show evidence of tuberculosis elsewhere.

It is known that several lesions may produce a ring or disc enhancing lesion. Tumours are known to do this ${ }^{10}$ and we had one such case. We do not think any of the present 39 harbours a tumour. In 23 the lesion has cleared and 10 others are clinically normal. Cysticercosis may produce a similar lesion but cysticercosis is rare in this part of India and is much more often multiple than single. None of our cases had 

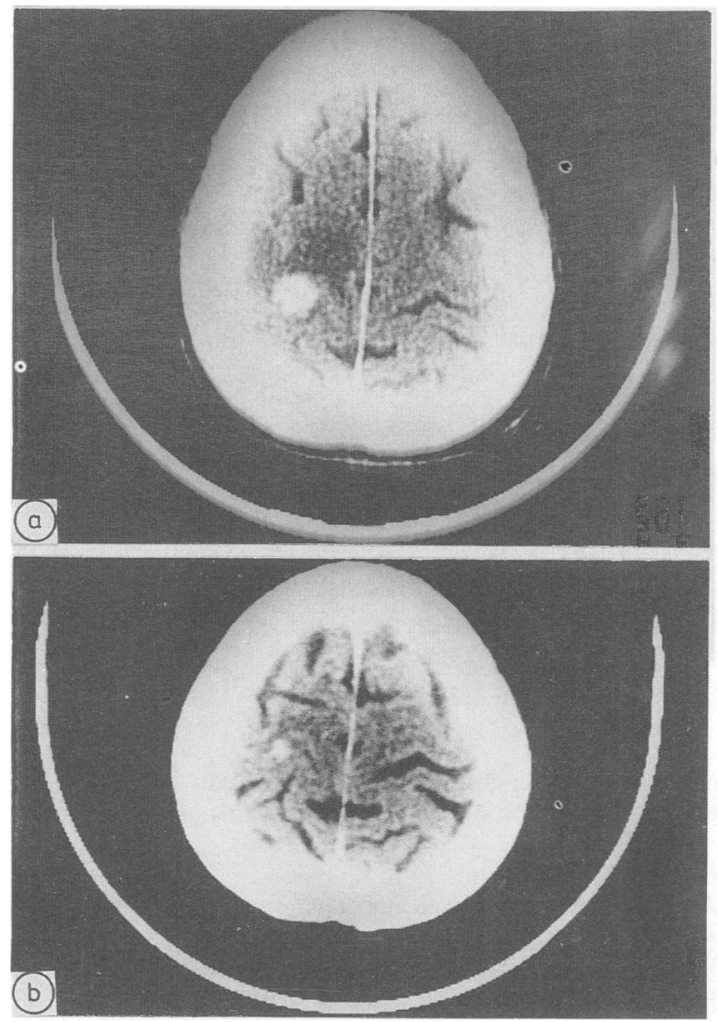

Fig 3 Gradual clearance of a ring or disc enhancing lesion on antitubercular therapy. (a) Disc enchancing lesion, (b) showing partial clearance 2 months later on antitubercular therapy.

peripheral evidence of cysticercosis and complete clearing without drugs would be most unlikely in such a high proportion of cases of cysticercosis. Rana et al studied CT findings in cysticercosis in India. In 62 cases there was no case with a solitary ring enhancing or disc lesion as described here (personal communication). Minguetti and Ferreira ${ }^{11}$ reporting on 171 cases from Brazil found only six which apparently showed features similar to our cases. Thus such a lesion is unusual in cysticercosis (approximately $3-4 \%$ ) and its resolution in 3 months even more unusual. Sethi et $\mathrm{l}^{9}$ raised the possibility that these lesions may represent a form of encephalitis. As mentioned before, in 12 of the 39 cases the fits were of greater than 3 months' duration, and in some more than 1 year, so that in these the encephalitis would have to be a subacute or chronic form and then clear with treatment. This seems most unlikely. It is possible that our cases of ring or disc enhancing lesion may represent two or more types of cases. Those of short duration (especially those with a cluster of fits at onset) may represent a focal encephalitic process. The rest could well represent other granulomatous lesions, most likely tuberculomas. However, one of the cases verified histologically and five of the 10 with tuberculosis elsewhere in the body had short duration epilepsy. In one case seen after this series was closed, fits appeared in a cluster while on treatment for miliary tuberculosis and the scan showed a ring or disc enhancing lesion which was resolving on CT scan after 2 months.

We find it difficult to resolve the dilemma of aetiology. Biopsy at first diagnosis seems unnecessary and unwarranted as the lesion clears with medical therapy. Watching without definite treatment may be hazardous especially if patient follow-up is defective. The only scientific way would be prospective randomised comparison of antituberculous therapy and anticonvulsants alone. Our experiences make us feel that this would not be practicable.

\section{References}

1 Commission on Classification and terminology of the International league against epilepsy. Proposal for revised clinical and electroencephalographic classification of epileptic seizures. Epilepsia 1981;22:489-501.

2 Gastaut H, Gastaut JL. Computerized transverse axial tomography in epilepsy. Epilepsia 1976;17:325-36.

3 Bogdanoff BM, Stafford CR, Green L, Gonzalex CF. Computerized trans axial tomography in the evaluation of patients with focal epilepsy. Neurology 1975;25:1013-7.

4 Collard M, Dupont H, Noel G. Computerized transverse axial tomography in epilepsy. Epilepsia 1976;17: 339-42.

5 Scollo-Lavizzari G, Eichhorn K, Wuthrich R. Computerized transverse axial tomography in the diagnosis of epilepsy. Eur Neurol 1977;15:5-8.

6 McGahan JP, Dublin AB, Hill RP. The evaluation of seizure disorders by computerized tomography. J Neurosurg 1979;50:328-32.

7 Yang J, Berger PE, Cohen ME, Duffner RK. Computed tomography and childhood seizure disorders. Neuro$\log y$ 1979;29:1084-8.

8 Bhargava S, Tandon PN. CNS tuberculosis-lessons learnt from CT studies. Neurology India 1980;28: 207-12.

9 Sethi PK, Kumar BR, Madan VS, Mohan V. Appearing and disappearing CT scan abnormalities and seizures. J Neurol Neurosurg Psychiatry 1985;48:866-9.

10 Lee SH, Rao KCVG. Primary tumors in adults. In: Lee SH, Rao KCVG, eds. Cranial Computed Tomography. New York: McGraw Hill Book Company, 1983:241-93.

11 Minguetti G, Ferriera MVC. Computed tomography in neurocysticercosis. J Neurol Neurosurg Psychiatry 1983;46:836-42. 